\title{
Développements apportés au logiciel OEDIPE de simulation de mesures anthroporadiamétriques pour la prise en compte des données biocinétiques dans la modélisation de la contamination interne
}

\author{
S. LAMART $^{1}$, L. DE CARLAN ${ }^{1}$, E. BLANCHARDON ${ }^{1}$, D. FRANCK ${ }^{1}$
}

(Manuscrit reçu le 14 mai 2007, accepté le 28 novembre 2007)

RÉSUMÉ Dans le cadre de l'amélioration des techniques d'étalonnage en anthroporadiamétrie, le Laboratoire d'Évaluation de la Dose Interne de l'IRSN a développé un outil numérique, « OEDIPE », pour modéliser la contamination interne dans des fantômes numériques voxélisés, simuler la mesure anthroporadiamétrique et estimer la dose interne. Si la précédente version d'OEDIPE permettait de bien modéliser des géométries de source comprenant un seul radionucléide localisé dans un organe particulier, elle était cependant limitée pour la modélisation d'une contamination formée d'un mélange de radionucléides dont la localisation évolue à partir de l'incorporation pour se distribuer dans plusieurs organes. Cet article a pour but de décrire les nouveaux modules développés pour permettre la définition de sources hétérogènes en composition et en répartition ainsi que l'importation automatique de distributions d'activité dérivées du calcul biocinétique. Ces nouveaux outils vont permettre d'étudier des cas de contamination plus complexes et l'influence de la biocinétique en mesure in vivo, afin de mieux estimer les coefficients d'étalonnage et les incertitudes correspondantes.

ABSTRACT Recent developments in the software package OEDIPE - enabling the simulation of in vivo counting - to use the biokinetic data in the modelling of the internal contamination. As part of the improvement of calibration techniques of in vivo counting, the Laboratory of Internal Dose Assessment of IRSN has developed a computer tool, "OEDIPE", to model internal contamination, to simulate in vivo counting and to calculate internal dose. The previous version of this software enabled to easily model sources made of a single radionuclide and located in a single organ but could not represent a mixture of radionuclides which distribution evolves from the time of intake according to the biokinetics of the radionuclides. The purpose of this article is to describe a new facility developed in the software to allow the modelling of sources heterogeneous in composition and in distribution and optionally to automatically integrate the distribution of the activity in different tissues estimated by biokinetic calculation at any time since the intake. These new developments give the opportunity to study much more complex contamination cases and the influence of the biokinetics on the in vivo counting, leading to a better assessment of the calibration coefficients and of the corresponding uncertainties.

Keywords: in vivo counting / voxel phantoms / Monte-Carlo simulation (MCNPX) / heterogeneous contamination / biokinetic models / OEDIPE

1 IRSN, Laboratoire d'Évaluation de la Dose Interne, DRPH/SDI/LEDI, B.P. 17, 92262 Fontenay-aux-Roses Cedex, France. 


\section{Introduction}

En cas de contamination interne, l'anthroporadiamétrie, ou mesure in vivo, permet d'identifier les radionucléides incorporés et d'évaluer la rétention d'activité correspondante. Les fantômes physiques anthropomorphes utilisés actuellement pour l'étalonnage des détecteurs servant à la mesure ne sont qu'une représentation imparfaite du corps humain tant du point de vue de l'anatomie que de la répartition de la contamination qui est modélisée de façon homogène. On en déduit donc des coefficients d'étalonnage parfois difficiles à appliquer aux cas réels de contamination. Une nouvelle technique d'étalonnage consiste à remplacer ces fantômes par des fantômes numériques voxélisés plus réalistes, basés sur des images médicales obtenues par IRM ou scanner. Dans ce cadre de l'amélioration des techniques d'étalonnage en anthroporadiamétrie et d'évaluation de la dose interne, le Laboratoire d'Évaluation de la Dose Interne (LEDI) a développé le logiciel de calcul OEDIPE (Outil d'Évaluation de la Dose Interne PErsonnalisée). Celui-ci permet, d'une part, de modéliser une mesure in vivo pour obtenir par calcul le spectre anthroporadiamétrique (Franck et al., 2003, 2001 ; Borisov et al., 2005b, 2002 ; Pierrat et al., 2005 ; de Carlan et al., 2007) et, d'autre part, d'obtenir la distribution de la dose (Aubineau-Lanièce et al., 2005a, 2005b) au niveau de la région contaminée, au moyen de fantômes numériques voxélisés et d'un code de calcul Monte-Carlo (MCNPX) du transport des particules dans la matière (de Carlan et al., 2005, 2004 ; Borisov et al., 2005a).

Une des difficultés rencontrées lors de la modélisation d'une mesure in vivo est de représenter la géométrie de la source. Si la précédente version d'OEDIPE permettait de bien modéliser des géométries de source comprenant un seul radionucléide localisé dans un organe particulier, elle était cependant limitée pour la modélisation d'une contamination formée d'un mélange de radionucléides dont la localisation évolue à partir de l'incorporation pour se distribuer dans plusieurs organes.

Cet article a pour but de montrer les nouveaux développements dédiés à la biocinétique. Un module a été ajouté à OEDIPE pour prendre en compte les données biocinétiques dans la modélisation de la source, afin de simuler l'évolution temporelle de la localisation de la contamination selon les prévisions des modèles biocinétiques de la Commission Internationale de Protection Radiologique (CIPR).

Le développement a été réalisé en trois étapes principales:

- La première a consisté à créer une interface entre un code de calcul biocinétique externe et OEDIPE afin d'obtenir les rétentions d'activité dans les compartiments définis par la CIPR puis de les associer aux organes segmentés 


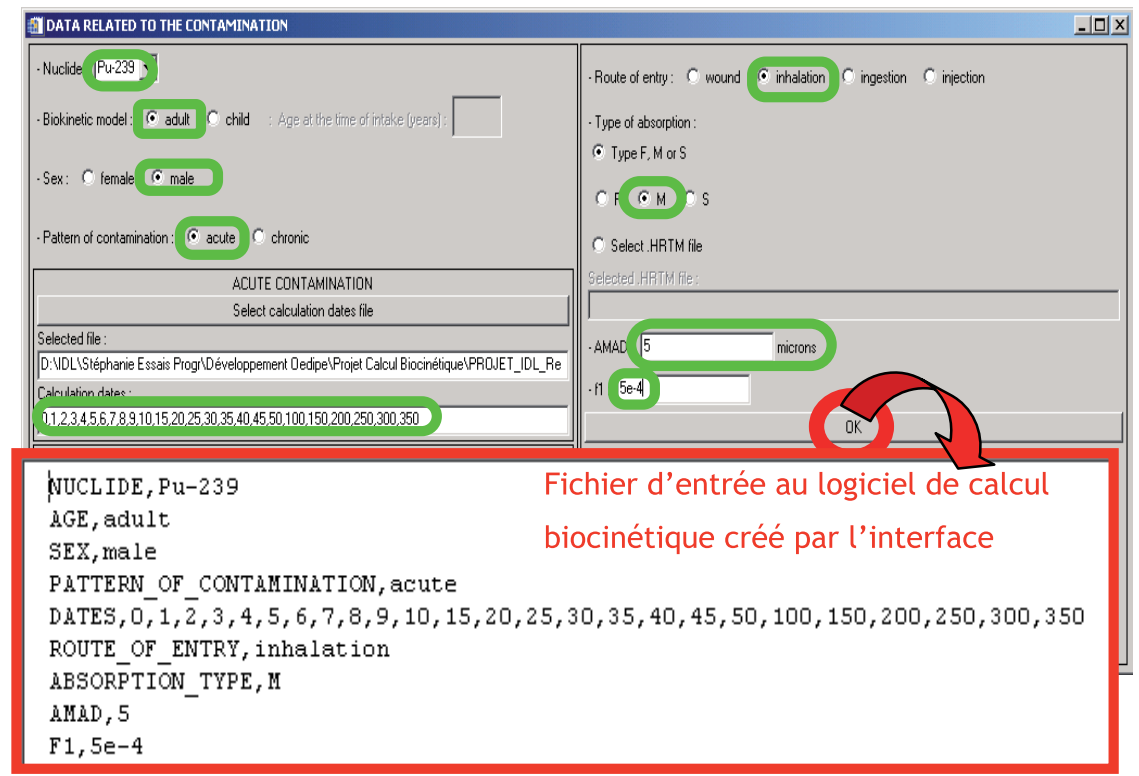

Figure 1 - Interface de création du fichier d'entrée au code de calcul biocinétique avec un exemple de fichier.

Interface to create the input file to the biokinetic calculation code with an example file.

du fantôme voxélisé et de donner une représentation graphique interactive de ces rétentions.

- Dans la deuxième étape, une interface de création de sources hétérogènes en composition isotopique et en répartition dans le corps a été ajoutée à OEDIPE. Ceci a nécessité la modification de l'algorithme de création du fichier d'entrée MCNPX, la version précédente n'étant pas adaptée à la description de sources hétérogènes.

- Enfin, les nouveaux développements ont été validés par la création de fantômes-sources reflétant la localisation des points-sources simulés.

\section{Développement d'une interface entre un code de calcul biocinétique et OEDIPE}

La première partie du travail consiste à calculer, à partir des conditions d'exposition (nature du radionucléide, activité incorporée, voie d'entrée, caractéristiques physico-chimiques...), les rétentions d'activité, en fonction du temps, dans les différents organes segmentés du fantôme numérique voxélisé créé sous OEDIPE. Les codes de calcul biocinétique estiment de telles rétentions dans 


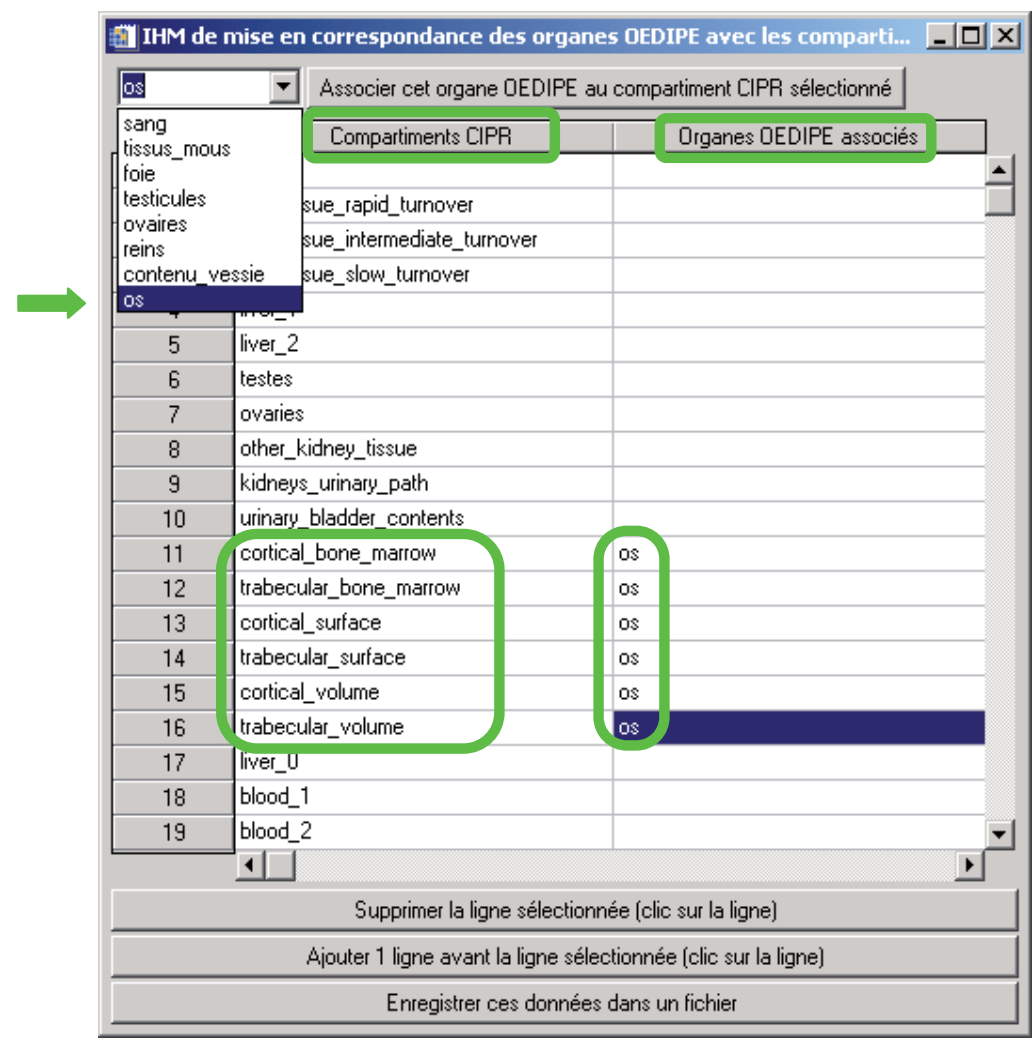

Figure 2 - Interface permettant d'associer à chaque compartiment du modèle biocinétique un organe du fantôme segmenté.

Interface enabling to assign to each compartment of the biokinetic model an organ of the voxel phantom.

les compartiments de modèles qui n'ont pas forcément une correspondance anatomique directe. Des interfaces sont donc développées pour permettre de :

- créer le fichier d'entrée au code de calcul biocinétique DCAL (Dose and risk CALculation, Oak Ridge National Laboratory, États-Unis (Eckerman et al., 2001)) définissant les conditions d'exposition (Fig. 1),

- mettre en correspondance les compartiments du modèle biocinétique avec les organes segmentés du fantôme (Fig. 2),

- calculer les activités dans les organes du fantôme voxélisé en fonction du temps, à partir du fichier de sortie du calcul biocinétique et de la correspondance entre compartiments du modèle et organes du fantôme. Cette interface donne également une représentation graphique conviviale de l'évolution de la contamination (Fig. 3). 


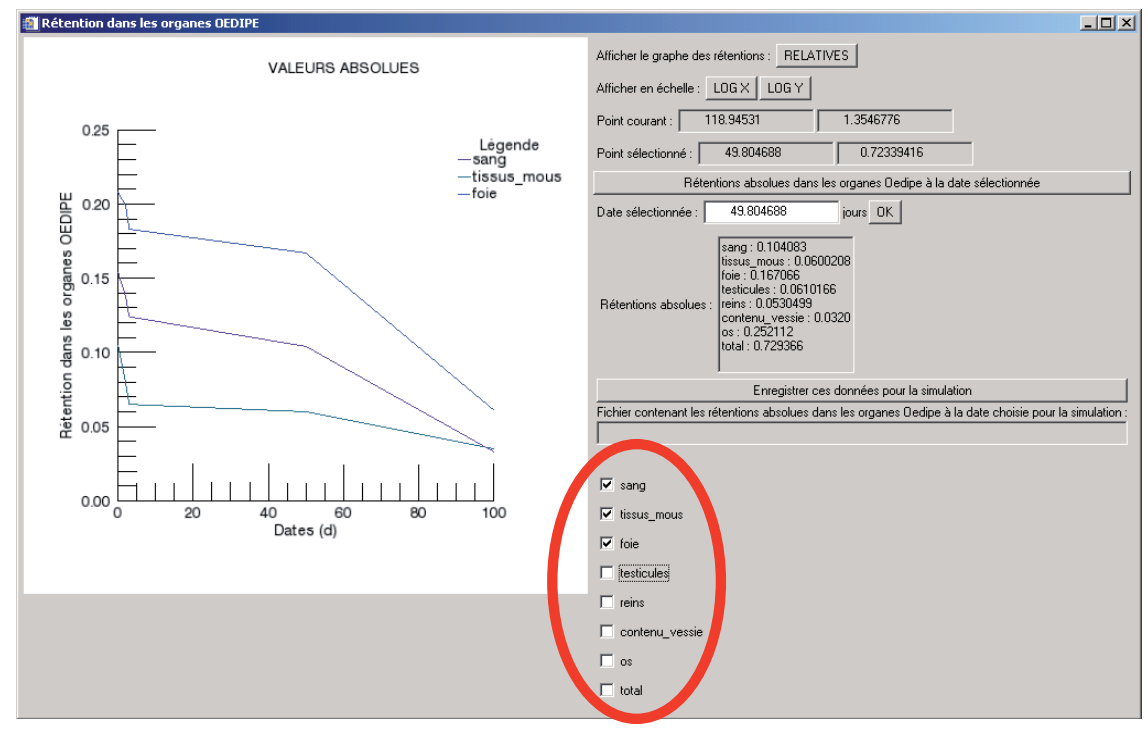

Figure 3 - Interface de visualisation de l'évolution des rétentions d'activité dans les organes du fantôme, permettant de choisir les dates pour lesquelles une simulation de mesure va être réalisée. Interface to visualise the evolution of activity in the organs of the phantom, enabling to choose the times for which a simulation of in vivo counting will be performed.

L'utilisateur visualise ainsi l'évolution de la localisation de la contamination dans les différents organes et peut choisir les dates auxquelles effectuer une simulation de la mesure anthroporadiamétrique.

\section{Développement dans OEDIPE d'outils de gestion d'une source hétérogène en répartition et en composition et réécriture de l'algorithme de création du fichier d'entrée au code de calcul MCNPX}

Il est alors nécessaire d'attribuer son activité à chaque organe-source du fantôme voxélisé. Or, il n'était possible jusqu'ici de définir dans OEDIPE qu'un unique organe-source pour la simulation des mesures in vivo. Une nouvelle interface, présentée en figure 4, a donc été développée pour créer interactivement une source hétérogène en répartition et en composition, en définissant les activités de chaque radionucléide dans chaque organe-source ou importer la distribution d'activité issue du calcul biocinétique pour l'attribuer automatiquement aux organes du fantôme voxélisé.

Ensuite, le logiciel crée automatiquement le fichier d'entrée au code de calcul Monte-Carlo. Pour pouvoir décrire cette distribution de source complexe, de 


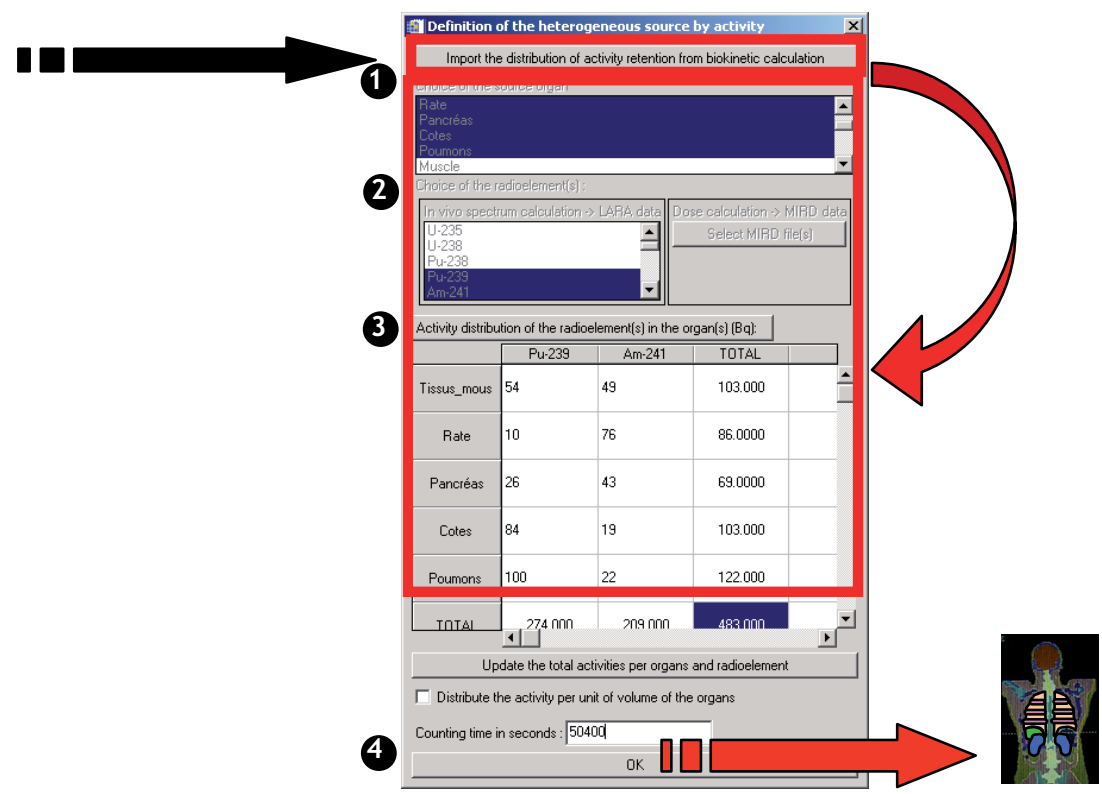

Figure 4 - Interface de création de sources hétérogènes en répartition et en composition dans le fantôme voxélisé avec possibilité d'importer la distribution d'activité dérivée du calcul biocinétique. 1: Choix de(s) l'organe(s) source, 2 : choix du ou des radionucléide(s), 3 : attribution des activités de chaque radionucléide à chaque organe-source, 4 : validation de la saisie de la source. Interface to create sources heterogeneous in composition and in distribution in the voxel phantom with an optional importation of the distribution of activity derived from the biokinetic calculation. 1: Choice of the source-organ, 2: choice of the radionuclide(s), 3: assignment of activities of each radionuclide in each source-organ, 4: validation of the inputs defining the source.

nombreuses modifications ont dû être apportées à l'algorithme de création du fichier d'entrée MCNPX en remplaçant l'ancien format de description de la géométrie du fantôme («voxel coupling ») (Franck et al., 2001) par une nouvelle description plus efficace et plus commode (« repeated structures ») (AubineauLanièce et al., 2005b) et en introduisant une nouvelle méthode pour spécifier les sources hétérogènes dans le fichier d'entrée au code de calcul.

\section{Validation des développements réalisés dans OEDIPE}

Suite à l'introduction de ces modifications dans le code d'OEDIPE, une validation a été réalisée par la création de fantômes sources. Un fantôme source est constitué par l'ensemble des points à partir desquels le code MCNPX a effectivement simulé l'émission d'une particule. Pour le créer, ces points sources sont enregistrés au cours du calcul MCNPX. Un programme a ensuite été implémenté pour 


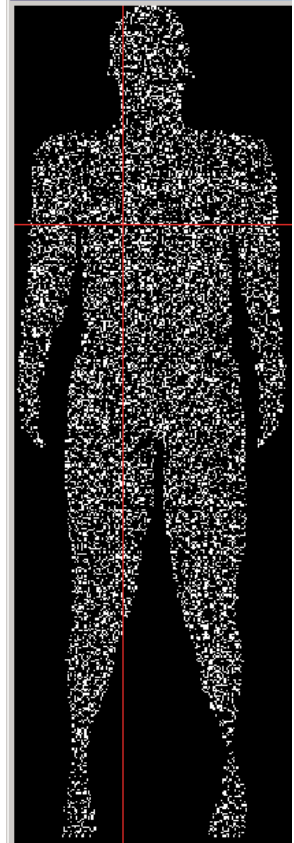

(a)

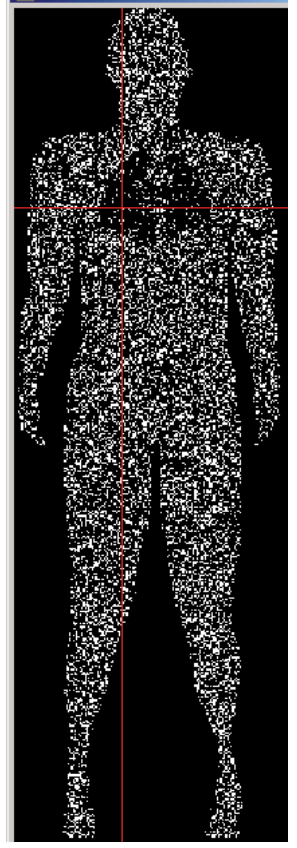

(b)

Figure 5 - Visualisation de fantômes sources créés à partir d'une répartition homogène de l'activité dans le corps entier du fantôme Zubal par unité de volume (a) et par unité de masse (b).

Visualisation of source-phantoms created from a homogeneous distribution of activity in the whole body of the Zubal voxel phantom per unit of volume (a) and per unit of mass (b).

transformer l'ensemble des positions des points en un fantôme numérique lisible par OEDIPE et fournissant la proportion de simulations de la transformation nucléaire de chaque radionucléide dans chaque organe. Des fantômes sources ont ainsi été réalisés pour contrôler la répartition de l'activité effectivement simulée par le code MCNPX à partir des instructions données dans le fichier d'entrée (Fig. 5).

La figure 5 montre qu'une répartition de l'activité par unité de masse met en évidence les poumons. En effet, leur densité est beaucoup plus faible (environ 0,3) que celle des autres tissus (de l'ordre de 1 ou supérieure). En revanche, une répartition homogène de l'activité par unité de volume produit effectivement une distribution régulière des points sources dans l'ensemble du volume du fantôme.

Une validation quantitative de la création d'une distribution de source à l'aide de l'interface présentée en figure 4 a été réalisée par la comparaison des 


\begin{tabular}{c|l|c}
\cline { 2 - 3 } & \multicolumn{1}{|c|}{ Cs-137 } & \multicolumn{1}{c}{ Co-60 } \\
\hline Foie & 0 & 63.5 \\
\hline Corps_Entier & 1000 & 1936.5 \\
\hline TOTAL & 1000.00 & 2000.00 \\
\hline
\end{tabular}

Figure 6 - Distribution d'activité hétérogène en composition et en répartition définie par l'utilisateur pour la validation de l'outil. Corps entier : activité à répartir dans tous les organes en proportion de leur masse. Foie : activité à ajouter dans l'organe spécifié.

Distribution of activity heterogeneous in composition and in distribution defined by the user in the validation of the tool. "Corps entier": whole body activity to be distributed in every organ in proportion of its mass. "Foie": liver activity to be added in the specified organ.

proportions de simulations de la transformation nucléaire de chaque radionucléide dans chaque organe. Pour cette validation, deux radionucléides sont distribués dans le fantôme numérique voxélisé à l'aide d'OEDIPE : 1000 Bq de Cs-137 sont répartis dans tous les organes du corps en proportion de leur masse et $2000 \mathrm{~Bq}$ de Co-60 sont répartis à raison de $5 \%$ de l'activité totale dans le foie et de $95 \%$ distribués proportionnellement à la masse des autres organes (Fig. 6).

À partir de la répartition définie figure 6, l'activité présente dans chaque organe pour chaque radionucléide est calculée par OEDIPE sur la base des densités des tissus. Ces valeurs (Tab. I) ont été validées par un calcul indépendant.

Ensuite, OEDIPE calcule les distributions à inscrire dans le fichier d'entrée au code de calcul MCNPX pour définir la source. Elles sont obtenues en multipliant chaque activité par l'intensité des raies d'émission énergétique du radionucléide considéré et en normalisant les valeurs. Le résultat du calcul a été vérifié.

Ces distributions sont réécrites dans le fichier de sortie du calcul MCNPX. Elles sont reproduites dans la colonne «Valeur attendue » du tableau II et comparées aux distributions effectivement échantillonnées au cours du calcul (colonne «Valeur échantillonnée »). Le rapport «Attendue/échantillonnée » est égal à 1 pour presque tous les organes et s'en écarte de moins de $2 \%$ pour la région extrathoracique, l'estomac et le pancréas.

Les mêmes contrôles ont été réalisés pour vérifier la proportion des photons émis par le Cs-137 et le Co-60 à l'intérieur de chaque organe. Les rapports «Attendue/échantillonnée » sont tous très proches de 1. Ces contrôles permettent donc d'affirmer que les distributions effectivement générées au cours du calcul Monte-Carlo reproduisent fidèlement la source hétérogène en répartition et en 
TABLEAU I

Distribution d'activité calculée par OEDIPE à partir de la source définie par l'utilisateur (Bq). Distribution of activity computed by OEDIPE from the source defined by the user (Bq).

\begin{tabular}{lcc}
\hline Organes & Cs-137 & Co-60 \\
\hline Tissus mous & 110 & 220 \\
Rate & 3,5 & 6,7 \\
\hline Pancréas & 0,5 & 0,9 \\
\hline Os & 150 & 280 \\
\hline Poumons & 9,5 & 19 \\
Muscle & 460 & 900 \\
Foie & 18 & 99 \\
Intestin & 30 & 58 \\
Sang & 13 & 25 \\
Urine & 3,9 & 7,5 \\
Graisse & 190 & 370 \\
Testicules & 0,9 & 1,8 \\
Estomac & 3,1 & 6,0 \\
Rein & 4,7 & 9,1 \\
\hline ET & 0,9 & 1,7 \\
Total & 1000 & 2000 \\
\hline
\end{tabular}

composition définie par l'utilisateur. Par ailleurs, l'importation des distributions dérivées du calcul biocinétique par le code DCAL pour la création de la source dans OEDIPE a été également validée (Lamart et al., 2007).

\section{Conclusion}

Dans le cadre de l'amélioration des méthodes d'étalonnage en mesure anthroporadiamétrique, le LEDI a développé le logiciel OEDIPE pour simuler la mesure en modélisant le sujet à mesurer et la distribution de la contamination interne. L'objectif du travail décrit ici était de définir la source de façon plus réaliste à partir de modèles biocinétiques, afin d'améliorer l'étalonnage des détecteurs utilisés pour la mesure et par conséquent l'estimation de la dose. Ainsi, le nouveau module développé permet de créer interactivement un fichier d'entrée pour un code de calcul biocinétique contenant les caractéristiques d'une exposition par contamination. Le fichier de sortie du code de calcul biocinétique est ensuite traité par d'autres interfaces pour relier les retentions calculées dans les compartiments d'un modèle biocinétique avec les activités dans les organes segmentés d'un fantôme voxélisé. Une représentation graphique, en fonction du 
TABLEAU II

Comparaison de la distribution échantillonnée au cours du calcul Monte-Carlo avec celle attendue par l'utilisateur. ET, région extrathoracique.

Comparison of the distribution of activity sampled during the run of Monte-Carlo calculation with the one expected by the user. ET, extrathoracic region.

\begin{tabular}{lccc}
\hline Organes & Valeur attendue & Valeur échantillonnée & Attendue / échantillonnée \\
\hline Tissus mous & $1,09 \times 10^{-1}$ & $1,09 \times 10^{-1}$ & 1,00 \\
Rate & $3,39 \times 10^{-3}$ & $3,38 \times 10^{-3}$ & 1,00 \\
Pancréas & $4,68 \times 10^{-4}$ & $4,72 \times 10^{-4}$ & 0,99 \\
Os & $1,43 \times 10^{-1}$ & $1,43 \times 10^{-1}$ & 1,00 \\
\hline Poumons & $9,27 \times 10^{-3}$ & $9,29 \times 10^{-3}$ & 1,00 \\
Muscle & $4,52 \times 10^{-1}$ & $4,52 \times 10^{-1}$ & 1,00 \\
Foie & $4,36 \times 10^{-2}$ & $4,36 \times 10^{-2}$ & 1,00 \\
Intestin & $2,91 \times 10^{-2}$ & $2,91 \times 10^{-2}$ & 1,00 \\
Sang & $1,28 \times 10^{-2}$ & $1,28 \times 10^{-2}$ & 1,00 \\
Urine & $3,78 \times 10^{-3}$ & $3,78 \times 10^{-3}$ & 1,00 \\
Graisse & $1,84 \times 10^{-1}$ & $1,84 \times 10^{-1}$ & 1,00 \\
Testicules & $8,84 \times 10^{-4}$ & $8,82 \times 10^{-4}$ & 1,00 \\
Estomac & $3,05 \times 10^{-3}$ & $3,03 \times 10^{-3}$ & 1,01 \\
Rein & $4,60 \times 10^{-3}$ & $4,58 \times 10^{-3}$ & 1,00 \\
ET & $8,51 \times 10^{-4}$ & $8,36 \times 10^{-4}$ & 1,02 \\
\hline
\end{tabular}

temps, de ces activités est fournie à l'utilisateur pour choisir la date de la simulation. Enfin, la distribution de l'activité dérivée du modèle biocinétique à une date donnée est reproduite dans le fantôme voxélisé pour simuler les mesures in vivo par calcul Monte-Carlo.

Les premières applications de ces développements sont actuellement réalisées dans le cadre de différents scénarios de contamination avec l'objectif d'évaluer l'influence de paramètres biocinétiques sur la mesure in vivo et la valeur de l'activité estimée, principalement par le calcul des coefficients d'étalonnage dans le cas de la contamination pulmonaire par des actinides. Les applications futures peuvent être prospectives, puisque la prévision de futures mesures in vivo peut aider à définir un programme de surveillance de l'exposition interne. Elles peuvent être également rétrospectives par la ré-évaluation des activités résultant d'une ancienne contamination et calculées précédemment à partir de la méthode d'étalonnage classique avec des fantômes physiques.

Remerciements. Ce travail est financé par le PIC DOSINTER, Programme d'Intérêt Commun entre AREVA NC et l'IRSN. 


\section{REFERENCES}

Aubineau-Lanièce I., de Carlan L., Clairand I., Lemosquet A., Chiavassa S., Pierrat N., Bardies M., Franck D. (2005a) Current developments at IRSN on computational tools dedicated to assessing doses for both internal and external exposure, Radiat. Prot. Dosim. 115, 522-529.

Aubineau-Lanièce I., de Carlan L., Pierrat N., Franck D., Chiavassa S., Bardies M. (2005b) Application of voxel phantoms for internal dosimetry at IRSN using a dedicated computational tool. In: Monte Carlo 2005 Topical Meeting, Chattanooga, TN.

Borisov N., Franck D., de Carlan L., Laval L. (2002) A new graphical user interface for fast construction of computation phantoms and MCNP calculations: application to calibration of in vivo measurement systems, Health Phys. 83, 272-279.

Borisov N., Franck D., De Carlan L., Pierrat N., Kochetkov O., Yatsenko V. (2005a) A new facility for MCNP application in whole body counting and internal dosimetry. In: Monte Carlo 2005 Topical Meeting, Chattanooga, TN.

Borisov N., Yatsenko V., Kochetkov O., Gusev I., Vlasov P., Kalistratova V., Nisimov P., Levochkin F., Borovkov M., Stolyarov V., Tsedish S., Tyurin I., Franck D., de Carlan L. (2005b) Checking a Monte Carlo utility for whole body counting during in vivo experiment. In: Monte Carlo 2005 Topical Meeting, Chattanooga, TN.

de Carlan L., Aubineau-Lanièce I., Diaz J.C., Jourdain J.R.J., Le Guen B., Pierrat N., Franck D. (2004) Potential of new imaging and calculation techniques for the activity and dose assessment in case of localized contamination. In: IRPA 2004, Madrid.

de Carlan L., Roch P., Blanchardon E., Franck D. (2005) New method of voxel phantom creation: application for whole-body counting calibration and perspectives in individual internal dose assessment, Radiat. Prot. Dosim. 116, 160-164.

de Carlan L., Roch P., Blanchardon E., Franck D. (2007) Application of Voxel Phantoms in WholeBody Counting for the Validation of Calibration Phantoms and the Assessment of Uncertainties, Radiat. Prot. Dosim. DOI: 10.1093/rpd/nc1117.

Eckerman K.F., Leggett R.W., Cristy M., Nelson C.B., Ryman J.C., Sjoreen A.L., Ward R.C. (2001) DCAL: User's Guide to the DCAL System, Report ORNL/TM-2001/190, Oak Ridge National Laboratory, USA.

Franck D., Laval L., Borissov N., Guillierme P., Bordy J.M. (2001) Development of voxelised phantoms using MCNP Monte Carlo code: Application to in vivo measurement, Radioprotection 36, 77-86.

Franck D., Borissov N., de Carlan L., Pierrat N., Genicot J.L., Etherington G. (2003) Application of Monte Carlo calculations to calibration of anthropomorphic phantoms used for activity assessment of actinides in lungs, Radiat. Prot. Dosim. 105, 403-408.

Lamart S., de Carlan L., Blanchardon E., Franck D. (2007) Automatic application of ICRP biokinetic models in voxel phantoms for in vivo counting and internal dose assessment, Radiat. Prot. Dosim. DOI: $10.1093 / \mathrm{rpd} / \mathrm{ncm} 345$.

Pierrat N., de Carlan L., Cavadore D., Franck D. (2005) Application of monte carlo calculation for the virtual calibration of a low-energy in vivo counting system, IEEE Trans. Nucl. Sci. 52, 13531358. 